\title{
Etiological Factors in Pediatric Pseudotumor Cerebri Cases
}

\author{
Hulya Ince ${ }^{1(\mathbb{D})}$ \\ ${ }^{1}$ Department of Pediatrics, Division of Pediatric Neurology, Private Medical Park Samsun Hospital,Samsun
}

Copyright@ Author(s) - Available online at https://dergipark.org.tr/en/pub/mbsjohs

Content of this journal is licensed under a Creative Commons Attribution-NonCommercial 4.0 International

License,

Received: 01 June 2021, Accepted: 10 August 2021, Published online: 31 August 2021

(C) Ordu University Institute of Health Sciences, Turkey, 2021

\begin{abstract}
Objective: Pseudotumor cerebri (PTC) is the presence of the signs and symptoms of intracranial pressure increase in the absence of a tumoral, structural or vascular mass-occupying lesion. This study aimed to investigate the etiological factors involved in childhood PTC cases.

Methods: Data of the patients presented to the Private Medical Park Samsun Hospital, pediatric neurology outpatient clinic with the preliminary diagnosis of PTC between June 2015 and December 2020 were retrospectively reviewed. Demographic data, presentation symptoms, neuro-ophthalmological examination findings, laboratory data, and radiological imaging (brain magnetic resonance imaging, magnetic resonance venography) findings. The female (i.e., Group 1) and male (i.e., Group 2) children were compared concerning the etiological factors.

Results: The study cohort included 45 patients. There were 24 patients in Group 1 (i.e., female children) and 21 patients in Group 2 (i.e., male children). Among the 45 patients, 41 (91.1\%) had an overt etiological factor. Mean age of the study patients were 9.06 year [2,5 months-18 years]. The most common symptom was headache (64.4\%). Two groups were similar concerning mean patient age, pubertal stage, and rates of vitamin B12 deficiency, iron deficiency anemia, vitamin D deficiency, abnormal brain magnetic resonance imaging, and magnetic resonance venography findings. However, the obesity rate was significantly higher in Group 2 than Group 1 (32,7\% vs. 12,5\%, p<0.05).

Conclusion: A thorough investigation concerning etiology is crucial in pediatric PTC cases regardless of patient gender. Timely investigations regarding etiology will lead to timely treatment of this clinical condition and prevent irreversible devastating complications such as permanent hearing loss.

Key words: Pseudotumor cerebri, Intracranial pressure, etiology
\end{abstract}

Suggested Citation: Ince H. Etiological Factors in Pediatric Pseudotumor Cerebri Cases. Mid Blac Sea Journal of Health Sci, 2021; 7(2):198-204

\section{Address for correspondence/reprints:}

Hulya Ince

Telephone number: +90 (532) 1354070

E-mail: drhulyaince@yahoo.com 


\section{Introduction}

Pseudotumor cerebri (PTC) is defined as the presence of the signs and symptoms of intracranial pressure increase in the absence of a tumoral, structural or vascular mass-occupying lesion (1). It is classified as idiopathic intracranial hypertension (IIH) or primary PTC and secondary PTS. While secondary PTC develops due to underlying clinical conditions leading to an increase in the intracranial pressure, the etiology of IIH is still unclear (2). The most important complication of PTC is irreversible visual loss. Therefore, its timely recognition and treatment by elimination of the underlying clinical conditions are crucial. This study aimed to investigate the etiological factors involved in childhood PTC cases.

\section{Methods}

This study followed the Helsinki Declaration, and informed consent was taken from the caregivers of all children. It was approved by the Ethical Review Committee of the Samsun Training and Research Hospital (101864/01.10.2020). Data of the patients presented to the Private Medical Park Samsun Hospital, pediatric neurology outpatient clinic with the preliminary diagnosis of PTC between June 2015 and December 2020 were retrospectively reviewed. These patients were subsequently diagnosed with
PTC as per the diagnostic criteria previously reported in the literature (Table 1) (3). A second lumbar puncture was performed in all patients to see the response to the treatment and to evaluate the need for additional treatment. The procedure was repeated for the third time in patients with high cerebrospinal fluid pressure. No patient required more lomber puncture.

Demographic data, presentation symptoms, neuroophthalmological examination findings, laboratory data (i.e., complete blood count, blood biochemistry, acute phase reactants, thyroid function tests, iron, vitamin B12 and D levels, thrombophilia panel, cortisol, and parathormone levels), radiological imaging (brain MRI, magnetic resonance venography) findings and data regarding treatment were retrieved from electronic patient folders. The female (i.e., Group 1) and male (i.e., Group 2) children were compared concerning the etiological factors. All patients had undergone funduscopic examination, Snellen visual acuity test, and Goldmann visual field testing. Children younger than twelve were considered prepubertal, while those older than twelve were considered pubertal or postpubertal. Patients with body weight at or above the 95th percentile of children of the same age and gender were considered obese.

Table 1. Daignostic criteria for PTC

\section{Criteria for PTC diagnosis}

\section{A Papilledema \\ B Normal neurological examination findings except for cranial nerve anomalies}

C Neuro-imaging:

-Typical patients (i.e., female and obese): Normal brain parenchyma with no hydrocephaly, mass or structural lesion and no abnormal meningeal contrast enhancement in the magnetic resonance imaging (MRI) with or without gadolinium injection

-Other patients: MRI or MR venography with or without gadolinium injection; computerized tomography with contrast medium injection can be performed if MRI is unavailable or contraindicated

D Normal cerebrospinal fluid (CSF) content

E High lumbar puncture opening pressure; $250 \mathrm{mmCSF}$ in adults and $280 \mathrm{mmCSF}$ in children (250 mmCSF if the child is not sedated or obese)

PTC diagnosis is 'definite' if the criteria A-E are all fulfilled.

$P T C$ diagnosis is 'probable' if the A-D criteria are fulfilled but the lumbar puncture opening pressures are lower than the levels required for a definitive diagnosis.

\section{Criteria for PTC diagnosis in patients without papilledema}

-In the absence of papilledema, the diagnosis is PTC if the criteria B-E are fully met and if there is unilateral or bilateral abducens muscle paralysis

-In the absence of both papilledema and abducens muscle paralysis, PTC diagnosis is considered if the criteria B-E are fully met and if 3 of the neuro-imaging critera mentioned below are met.

\begin{tabular}{ll}
\hline i. & Empty sella \\
\hline ii. & Posterior ball flattening \\
\hline iii. & Distention of the perioptic subarachnoid space (optic nerve tortuosity may accompany may be present or absent) \\
\hline iv. & Transverse venous sinus stenosis \\
\hline
\end{tabular}




\section{Statistical analysis}

The Statistical Analysis for Social Sciences Software (SPSS v17.0, Chicago, IL, US) was used for all statistical analyses. Descriptive statistics were used, and data were given as percentages (\%) and means \pm standard deviations. The $\mathrm{p}$ value was considered significant when it was lower than 0,05 .

\section{Results}

A retrospective review of patient data revealed that 58 patients were evaluated during the study period with the provisional diagnosis of IIH. However, three patients with brain tumors and ten patients with normal CSF pressures were excluded. Therefore, the study cohort included 45 patients. There were 24 patients in Group 1 (i.e., female children) and 21 patients in Group 2 (i.e., male children). Among the 45 patients, 41 (91.1\%) had an overt etiological factor. Two patients in Group 1 $(8,3 \%)$ and one patient in Group $2(4,8 \%)$ had utterly typical results, and these patients were diagnosed with primary PTC. The etiological factors are presented in Table 2.

Mean age of the study patients was 9.06 year [2,5 months-18 years]. Two groups were similar regarding mean patient age (9,3 years in Group 1 [2,5 months18 years] and 8,7 years in Group 2 [9 months-16 years]).

Ten (22\%) patients were obese. Among these patients, $3(12.5 \%)$ were in Group 1, and 7 (32.7\%) were in Group 2. There was a significant difference between the two groups in this regard $(p<0.05)$.

Among 45 study patients, 28 were pubertalpostpubertal, while 17 were prepubertal. In Group 1, $66,6 \%$ of the patients were prepubertal, and $33,3 \%$ were pubertal, while in Group 2, 57\% of the patients were prepubertal, and $43 \%$ were pubertal. There was no significant difference between the groups concerning the pubertal stage.

The most common symptom was headache (64.4\%). Our analysis revealed that $62,5 \%$ of the patients in Group 1 and 66,6\% of Group 2 presented with headaches. The chief complaints of the patients who did not present with a headache but were diagnosed with PTC are displayed in Table 3.

A review of the patients' visual fields (VF) revealed that $21(46,6 \%)$ patients had narrowing of the VF. Seven patients $(15,5 \%)$ had normal VF. The VF tests could not be performed in $17(37.7 \%)$ cases. In Group 1, 11 patients had narrowing of the VF, four patients had normal VF, and 9 patients could not undergo VF testing. In Group 2, 10 patients had narrowing of the VF, 3 patients had normal VF, while 8 patients could not undergo VF testing. The two groups were not different regarding the results of $\mathrm{VF}$ assessments. In both groups, 1 patient with VF narrowing was diagnosed with PTC in the absence of headache. All patients except for 4 aged younger than 18 months $(91.1 \%)$ had bilateral papilledema.

Mean CSF pressure was 33,2 mmCSF [25-60] in the entire cohort. Fourteen $(31,1 \%)$ patients underwent lumbar puncture once, while $21(46.6 \%)$ underwent it twice, 7 underwent for three times, and $3(6.6 \%)$ underwent for four times.

A review of the laboratory data revealed that 10 $(22.2 \%)$ patients had normal vitamin levels while 35 $(77,8 \%)$ patients had a deficiency of one or more types of vitamins. In Group 1, 11 patients had vitamin B12 deficiency, 7 patients had iron deficiency anemia, and 16 patients had vitamin D deficiency. In Group 2, 12 patients had vitamin B12 deficiency, 6 had iron deficiency anemia, and 15 had low vitamin $\mathrm{D}$ levels. The two groups were similar in this regard.

The brain MRIs showed anomalies in 7 (29.1\%) patients in Group 1 and 6 (28.5\%) patients in Group 2.

In Group 1, two patients had arachnoid cysts, 4 had mastoiditis, and 1 patient had a slit ventricle and a cyst anterior to the mesencephalon. In Group 2, one patient had cortical atrophy, three patients had mastoiditis, one patient had pituitary adenoma, and one patient had pansinusitis. The two groups were not statistically different in this regard.

All MRV images were reviewed, and this analysis revealed that $19(42.2 \%)$ patients had abnormal MRV findings. In Group 1, 11 (45.8\%) patients and Group $2,8(38.1 \%)$ patients had sinus vein thrombosis. There was a significant difference between the two groups regarding the rates of abnormal MRV findings $(\mathrm{p}<0.05)$.

In patients with sinus vein thrombosis, all potential prethrombotic risk factors were investigated. The results of this analysis are displayed in Table 4. This analysis revealed that some of these patients had multiple risk factors.

All patients were given acetazolamide (10-30 $\mathrm{mg} / \mathrm{kg} /$ day) as first-line medical treatment. Patients with persistently high pressures at the post-treatment LP were given topiramate $(2-3 \mathrm{mg} / \mathrm{kg} /$ day $)$ treatment and furosemide $(1-2 \mathrm{mg} / \mathrm{kg} /$ day $)$ if topiramate treatment fails. In Group 1, $12(50 \%)$ patients were given acetazolamide treatment, $11(45.8 \%)$ patients received acetazolamide and topiramate treatments, while $1(4.1 \%)$ patient was given acetazolamide, topiramate, and furosemide treatments. Eleven patients received low molecular weight heparin (1 $\mathrm{mg} / \mathrm{kg}$, twice a day). In Group 2, 9 (42.8\%) patients were given acetazolamide treatment, 8 (38\%) patients 
received acetazolamide and topiramate treatments, while $4(19 \%)$ patients were given acetazolamide, topiramate, and furosemide treatments. Eight patients received low molecular weight heparin treatment.
None of the study participants were given steroid treatment nor surgical treatment.

Table 2. Etiological factors

\begin{tabular}{llll}
\hline Etiology & Group 1 $(\mathbf{n}=\mathbf{2 4})$ & Group 2 $(\mathbf{n}=\mathbf{2 1})$ & Total $(\mathbf{n}=\mathbf{4 5})$ \\
\hline Sinus vein thrombosis & $11(\% 45.8)$ & $8(\% 38)$ & $19(\% 42.2)$ \\
\hline Hypovitaminosis D & $2(\% 8.3)$ & $7(\% 32.7)$ & $9(\% 20)$ \\
\hline Hypervitaminosis D & - & $1(\% 4.72)$ & $1(\% 2.2)$ \\
\hline Hypervitaminosis A & $1(\% 4.16)$ & - & $1(\% 2.2)$ \\
\hline Miller-Fisher syndrome & $1(\% 4.16)$ & - & $1(\% 2.2)$ \\
\hline Hypovitaminosis B12 and D & $3(\% 12.5)$ & $3(\% 14.2)$ & $6(\% 13.3)$ \\
\hline $\begin{array}{l}\text { Low serum iron level and } \\
\text { hypovitaminosis B12 and D }\end{array}$ & $3(\% 12.5)$ & $1(\% 4.72)$ & $4(\% 8.8)$ \\
\hline Idiopathic & $3(\% 12.54)$ & $1(\% 4.72)$ & $4(\% 8.8)$ \\
\hline
\end{tabular}

Table 3. Chief complaints of the patients who did not present with headache

\begin{tabular}{llll}
\hline Complaints & Group 1 $(\mathbf{n}=\mathbf{2 4})$ & Group 2 $(\mathbf{n = 2 1})$ & Total $(\mathbf{n = 4 5})$ \\
\hline Strabismus or diplopia & $4(16.6 \%)$ & $3(14.28 \%)$ & $7(15.5 \%)$ \\
\hline Gait disturbances & $1(4.16 \%)$ & $1(4.76 \%)$ & $2(4.4 \%)$ \\
\hline Macrocephaly & - & $2(9.5 \%)$ & $2(4.4 \%)$ \\
\hline Bulging fontanelle & - & $1(4.76 \%)$ & $1(2.2 \%)$ \\
\hline Seizure & $1(4.16 \%)$ & - & $1(2.2 \%)$ \\
\hline Vomiting & $1(4.16 \%)$ & - & $1(2.2 \%)$ \\
\hline
\end{tabular}

Table 4. The prothrombotic risk factors determined in patients with sinus vein thrombosis

\begin{tabular}{llll}
\hline Group 1 (n=24) & \multicolumn{2}{l}{ Group 2 $(\mathbf{n}=\mathbf{2 1})$} \\
\hline Hyperhomocysteinemia & 1 & Hiperhomocysteinemia & 1 \\
\hline PAI-1 heterozygote & 1 & High lipoprotein a level & 1 \\
\hline MTHFR homozygote & 1 & PAI-1 and MTHFR heterozygote & 1 \\
\hline MTHFR homozygote + FV Leiden heterozygote & 1 & $\begin{array}{l}\text { PAI-1 and MTHFR heterozygote+Protein S } \\
\text { deficiency }\end{array}$ & 1 \\
\hline MTHFR and PAI-1 homozygote+FV Leiden heterozygote & 1 & MTHFR heterozygote+ Protein S deficiency & 1 \\
\hline FMF variant (Trabss syndrome) & 1 & Mastoiditis & 3 \\
\hline MTHFR heterozygote+Antithrombin III deficiency & 1 & & 8 \\
\hline Mastoiditis & 4 & & \\
\hline Total & 11 & & \\
\hline
\end{tabular}

PAI-1: Plasminogen activator inhibitor-1

MTHFR: Methylenetetrahydrofolate reductase

FV Leiden: Faktor V Leiden mutation

FMF: Familial Mediterrenean Fever

\section{Discussion}

Although PTC is usually called IIH, it is not usually "idiopathic". It is termed secondary PTC if an etiological factor is determined and primary PTC or IIH if an overt underlying reason is not detected (4). Loss of vision is its most serious complication of both primary and secondary PTC. On the other hand, the etiopathogenesis of secondary PTC has not been clearly understood yet, and it is more common in children than in adults (5). The most frequent causes of PTC are summarized in Table 5 (6). It was hypothesized that the mineralocorticoid receptors in the choroid plexus were activated, sodium-potassium ATPase pumps were stimulated, and subsequently, the CSF secretion and CSF pressure were both increased (7). It was reported that non-obese, young and male patients frequently had overt etiological reasons (8). While overt etiological factors were not usually identified in adults, they can be detected in $53-77 \%$ of the pediatric patient population (4). In our study, $91,1 \%$ had specific etiological factors, and this rate is higher than the rates reported in the literature. We believe that our study's relatively low mean patient age contributed to this difference.

Although PTC is primarily diagnosed in fertileaged women, it can be encountered at any age (9). Obesity is considered a risk factor. It was suggested that an increase in the obesity incidence led to an increase in the PTC rate in our country (10). However, most of the post pubertal (i.e., older than 12) children with PTC have risk factors such as obesity and female gender, the rate of obesity is relatively lower, and there is an even gender distribution in prepubertal children. While PTC 
incidence is $1 / 100000$, this rate increases 13 to 15 fold if there is $10 \%$ weight gain and 19 -fold if there is $20 \%$ weight gain (11). A study reported that the rate of obesity was $43 \%$ in PTC cases aged between 3 and 11 and $91 \%$ in those aged between 12 and 18 (10). The same study also concluded that the association of obesity and PTC got stronger with increasing age. It was also reported that the rate of PTC got higher among older women than those at younger ages. In our study, there was a relatively lower rate of obesity in prepubertal children. This finding was consistent with the literature.

The most common symptom of PTC is headache; it is encountered in $84-92 \%$ of these patients (12). Although its features, such as being diffuse, getting more severe at night, suggest that it is associated with the tension of the meninges and cerebral veins, its characteristics are not specific to PTC. Several studies reported in the literature stated that characteristics of the headache might vary, but it was usually a chronic daily, an acute repetitive, or a chronic repetitive type of headache. In our cohort, the rate of headache was $64,4 \%$. This rate is lower than the rates reported in the literature. This difference can be explained by the fact that most of our patients were at prepubertal age, and some of them were so young that they could not express their complaints. Visual complaints such as blurred vision, strabismus, diplopia, and decreased visual acuity constitute the second group of complaints in these patients (13). Temporary visual complaints accompany the clinical picture in $68-72 \%$ of the patients. It is known that the types and severities of the temporary visual complaints are not correlated with the severity of PTC, and they do not predict visual loss (14). It was reported that visual complaints were more dominant in patients with prepubertal PTC (15). Also, it was stated that $12-22 \%$ of the PTC cases were asymptomatic, and they were diagnosed incidentally by the detection of papilledema (16). This fact highlights the importance of a careful funduscopic examination. Bilateral papilledema is the most common finding in symptomatic cases ((17). It is detected in $86-100 \%$ of these patients.

Assessment of the VF provides essential data regarding PTC in children who can cooperate; however, it should be considered that this assessment is not easy (6). In our study, $46 \%$ of the patients had VF defects, and $91 \%$ had bilateral papilledema. Nausea, vomiting, dizziness, bulging of the fontanelles, seizure, tinnitus, gait disturbances, macrocephaly, and behavioral disorders are the other potential manifestations of PTC. In our study, one patient presented with vomiting, one with seizure, two with macrocephaly, one with fontanelle swelling, and two with gait disturbance.

Recently, critical values were determined for CSF opening pressures (3). Contrary to the old criteria, the new criteria did not include clinical signs and symptoms of intracranial pressure increase, and they consider some radiological findings as specific for PTC. As per these new criteria, a CSF opening pressure of higher than $28 \mathrm{cmH} 2 \mathrm{O}$ is considered positive for obese and sedated patients, while the threshold was $25 \mathrm{cmH} 2 \mathrm{O}$ for non-obese and nonsedated patients (3). On the other hand, in a study that considered CSF pressures in the range of 21-27 $\mathrm{cmH} 2 \mathrm{O}$ as significant, the authors noted that the opening pressure could be affected by the depth of sedation during lumbar puncture, excessive hyperventilation, pain, and stress (8). This study concluded that the CSF opening pressure should be measured again in patients whose opening pressures were normal, but the clinical pictures were consistent with PTC (8). In our study, the mean CSF opening pressure was 33,2 mmCSF, and this figure was consistent with the literature.

There are several reported regarding the association between infections and PTC $(18,19)$. These reports state that the reason for relatively higher rates of PTC in winter might be related to higher rates of respiratory viral infections in this season. Also, it was denoted that sinus vein thrombosis detected in $9-14 \%$ of the patients with PTC could be associated with a middle ear infection or mastoiditis $(18,19)$. On the other hand, it was suggested that hypervitaminosis A could lead to PTC via mineralocorticoid pathways (20). While some studies suggest that hypervitaminosis and hypovitaminosis $\mathrm{D}$ could be the underlying reason for PTC, some suggest that they can accompany PTC without a cause-and-effect relation (21). Anemia is another potential culprit, as per the published literature (22). It was stated that the tissue hypoxia stemming from anemia could lead to cerebral hemodynamic changes, increase the capillary permeability and cause an increase in the intracranial pressure. Iron deficiency anemia, megaloblastic anemia, and hemoglobinopathies are associated with increased risk of PTC (22). In this study, we detected sinus vein thrombosis, anemia, mastoiditis, hypervitaminosis $\mathrm{A}$ and $\mathrm{D}$, and hypovitaminosis $\mathrm{D}$ in our patients.

Brain MRI and MRV are essential diagnostic methods in PTC cases (6). They are used for diagnosing or eliminating neoplastic, vascular, or structural mass occupying lesions, sinus vein thrombosis, and other potential causes of secondary 
PTC. In our study, all patients had undergone contrast enhanced MRG and MRV. These imaging methods showed sinus vein thrombosis in $42,2 \%$ and abnormal MRI findings in $28,8 \%$ of the patients. The abnormal MRI findings included arachnoid cyst, mastoiditis, slit ventricle, anterior mesencephalon cyst, cortical atrophy, pituitary adenoma, and pansinusitis. Our study did not investigate the secondary radiological findings, and we consider this fact a weakness of our report.

The treatment regimens for PTC vary from center to center (23). The aim of treatment is to lower the intracranial pressure, prevent visual loss, and relieve headaches. Acetazolamide is the first-line medical treatment agent. It inhibits the carbonic anhydrase enzyme, decreases CSF production in the choroid plexus, and reduces pressure. The other medications are topiramate, furosemide, and steroids. The surgical treatment methods include lumboperitoneal and ventriculoperitoneal shunts, endoscopic fenestration of optic nerve sheaths, and stenting of dural sinuses. Although the standard approach is giving medical treatment first and saving the surgical treatment methods for medical treatment-unresponsive cases, surgical treatments can be considered the first-line treatment in patients with progressive and severe visual impairment. It was reported that $37-76 \%$ of patients responded to acetazolamide treatment (12). In a study including 31 pediatric patients with PTC, $19 \%$ had a permanent visual loss (24). Two studies reported from our country reported permanent visual loss rates of $11 \%$ and $7,1 \%(25,26)$.

These studies reported an acetazolamide treatment response rate of $46 \%$. In our study, we did not perform any surgical treatments, and none of our patients experienced permanent visual loss.

\section{Conclusion}

We believe that this result is due to comprehensive investigations regarding etiology performed at an early stage. Our findings indicate that a thorough investigation concerning etiology is crucial in pediatric PTC cases, regardless of patient gender. Timely investigations regarding etiology will lead to timely treatment of this clinical condition and prevent irreversible devastating complications such as permanent hearing loss.
Ethics Committee Approval: It was approved by the Ethical Review Committee of the Samsun Training and Research Hospital (101864/01.10.2020).

Peer-review: Externally peer-reviewed.

\section{Author Contributions:}

Concept, Design, Literature search, Data Collection and Processing, Analysis or Interpretation, Writing H.I.

Conflict of Interest: No conflict of interest was declared by the authors.

Financial Disclosure: The authors declared that this study hasn't received no financial support.

\section{References}

1. Masri A, Jaafar A, Noman R, Gharaibeh A, Ababneh $\mathrm{OH}$. Intracranial hypertension in children: Etiologies, clinical features, and outcome. J Child Neurol .2015;30(12):1562-8.

2. Standridge SM. Idiopathic intracranial hypertension in children: A review and algorithm. Pediatric Neurology. Pediatr Neurol; 2010;43: 377-90.

3. Friedman DI, Liu GT, Digre KB. Revised diagnostic criteria for the pseudotumor cerebri syndrome in adults and children Neurology. Neurology; 2013;81:1159-65.

4. Spennato P, Ruggiero C, Parlato RS, Buonocore MC, Varone A, Cianciulli E, et al. Pseudotumor cerebri. Child's Nervous System. Childs Nerv Syst; 2011;27:215-35.

5. Cleves-Bayon C. Idiopathic Intracranial Hypertension in Children and Adolescents: An Update. Headache .2018;58(3):485-93.

6. Phillips PH, Sheldon CA. Pediatric Pseudotumor Cerebri Syndrome. Journal of neuroophthalmology: the official journal of the North American Neuro-Ophthalmology Society. J Neuroophthalmol; 2017;37:33-40.

7. Salpietro V, Polizzi A, Bertè LF, Chimenz R, Chirico V, Ferraù V, et al.,Idiopathic intracranial hypertension: a unifying neuroendocrine hypothesis through the adrenal-brain axis. Neuroendocrinol Lett 2012; 33(6): 101-5.

8. Tibussek D, Distelmaier F, Karenfort M, Harmsen S, Klee D, Mayatepek E. Probable pseudotumor cerebri complex in 25 children. Further support of a concept. Eur J Paediatr Neurol .2017;21(2):2805.

9. Tibussek D, Schneider DT, Vandemeulebroecke $\mathrm{N}$, Turowski B, Messing-Juenger M, Willems PHGM, et al. Clinical spectrum of the pseudotumor cerebri complex in children. Child's Nerv Syst .2010;26(3):313-21. 
10.Balcer LJ, Liu GT, Forman S, Pun K, Volpe NJ, Galetta SL, et al. Idiopathic intracranial hypertension: Relation of age and obesity in children. Neurology .1999;52(4):870-2.

11.Friedman DI, Jacobson DM. Idiopathic intracranial hypertension. Journal of NeuroOphthalmology. J Neuroophthalmol; 2004;24:138-45.

12. Per H, Canpolat M, G um us H, Poyrazoĝlu HG, Yikilmaz A, Karak uc uk S, et al. Clinical spectrum of the pseudotumor cerebri in children: Etiological, clinical features, treatment, and prognosis. Brain Dev .2013;35(6):561-8.

13. Wall M, Kupersmith MJ, Kieburtz KD, Corbett JJ, Feldon SE, Friedman DI, et al. The idiopathic intracranial hypertension treatment trial clinical profile at baseline. JAMA Neurol 2014;71(6):693-701.

14. Corbett JJ, Savino PJ, Thompson HS, Kansu T, Schatz NJ, Orr LS, et al. Visual Loss in Pseudotumor Cerebri: Follow-up of 57 Patients from Five to 41 Years and a Profile of 14 Patients with Permanent Severe Visual Loss. Arch Neurol .1982;39(8):461-74.

15.Oguz MM, Polat E. Pediatric Pseudotumor Cerebri Syndrome with Etiological and Clinical Features. Firat University Medical Journal of Health Sciences. 2019; 33 (1): 15-9.

16.Goksan B. A guidebook on the approach to neurological diseases in children and adolescents. 2015. The child and adolescent neurology study group. Editors: Aysin DERVENT, Semih AYTA, Ozlem COKAR. pp 371-4.

17.Distelmaier F, Sengler U, Messing-Juenger M, Assmann B, Mayatepek E, Rosenbaum T. Pseudotumor cerebri as an important differential diagnosis of papilledema in children. Brain Dev. 2006;28(3):190-5.

18.Distelmaier F, Tibussek D, Schneider DT, Mayatepek E. Seasonal variation and atypical presentation of idiopathic intracranial hypertension in pre-pubertal children. Cephalalgia .2007;27(11):1261-4.

19.Lin A, Foroozan R, Danesh-Meyer H V., De Salvo G, Savino PJ, Sergott RC. Occurrence of Cerebral Venous Sinus Thrombosis in Patients with Presumed Idiopathic Intracranial Hypertension.Ophthalmology. 2006;113(12):2281-4.

20. Benzimra JD, Simon S, Sinclair AJ, Mollan SP. Sight-Threatening pseudotumour cerebri associated with excess vitamin a supplementation. Pract Neurol .2015;15(1):72-3.
21.Lessell S. Pediatric pseudotumor cerebri (idiopathic intracranial hypertension). Survey of Ophthalmology. Surv Ophthalmol; 1992;37:15566.

22.Henry M, Driscoll MC, Miller M, Chang T, Minniti CP. Pseudotumor cerebri in children with sickle cell disease: a case series. Pediatrics 2004;113: 265-69.

23.Avery RA, Shah SS, Licht DJ, Seiden JA, Huh JW, Boswinkel J, et al. Reference Range for Cerebrospinal Fluid Opening Pressure in Children. N Engl J Med .2010;363(9):891-3.

24.Gospe SM, Bhatti MT, El-Dairi MA. Anatomic and visual function outcomes in paediatric idiopathic intracranial hypertension. $\mathrm{Br} \quad \mathrm{J}$ Ophthalmol .2016;100(4):505-9.

25.Değerliyurt A, Teber S, Karakaya G, G uven A, Seker ED, Arhan EP, et al. Pseudotumor cerebri/idiopathic intracranial hypertension in children: An experience of a tertiary care hospital. Brain Dev .2014;36(8):690-9.

26.Gocmen R, Konuskan B. Clinical and neurogoral findings of pseudotic umor cerebr in children. Kirikkale University Faculty of Medicine Journal. 2018 Apr $30 ; 20(1): 33-40$. 